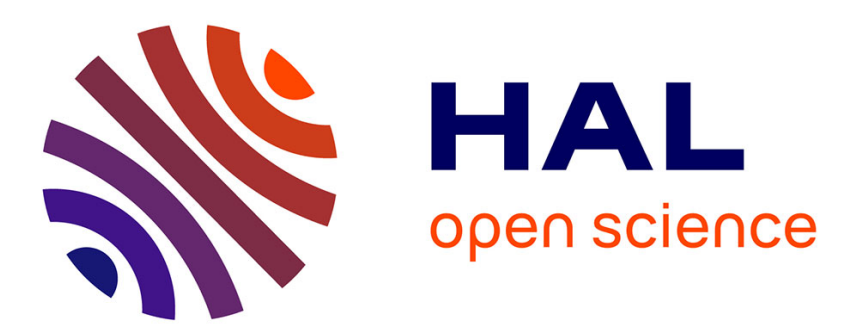

\title{
Photothermal beam deflection experiment applied to the measurement of directional optical properties of materials
}

\author{
J. Le Gall, P. Guinet, M. Olivier, J. Le Bris, C. Fort
}

\section{- To cite this version:}

J. Le Gall, P. Guinet, M. Olivier, J. Le Bris, C. Fort. Photothermal beam deflection experiment applied to the measurement of directional optical properties of materials. Journal de Physique IV Proceedings, 1994, 04 (C7), pp.C7-651-C7-654. 10.1051/jp4:19947154 . jpa-00253213

\section{HAL Id: jpa-00253213 https://hal.science/jpa-00253213}

Submitted on 1 Jan 1994

HAL is a multi-disciplinary open access archive for the deposit and dissemination of scientific research documents, whether they are published or not. The documents may come from teaching and research institutions in France or abroad, or from public or private research centers.
L'archive ouverte pluridisciplinaire HAL, est destinée au dépôt et à la diffusion de documents scientifiques de niveau recherche, publiés ou non, émanant des établissements d'enseignement et de recherche français ou étrangers, des laboratoires publics ou privés. 


\title{
Photothermal beam deflection experiment applied to the measurement of directional optical properties of materials
}

\author{
J. Le Gall, P. Guinet*, M. Olivier, J.Y. Le Bris and C. Fort \\ Centre d'Etudes Scientifiques et Techniques d'Aquitaine, CEA-CESTA, 33114 Le Barp, France \\ * Laboratoire d'Electronique de Technologie et d'Instrumentation, CEA-CENG, 38041 Grenoble, France
}

\begin{abstract}
A photothermal deflection experiment with a variable angle pump beam is presented. It is shown that directional absorptivity of rough or corrugated opaque samples can be measured by this technique. As an illustration the absorptivity in $p$ an s polarization of a $2 D$ surface-relief silicon grating is studied. Grating anomalies are detected and qualitatively discussed in the framework of an electromagnetic model.
\end{abstract}

\section{1 - INTRODUCTION}

When a parallel and monochromatic beam of light interacts with a material, a certain fraction $P_{a b s}$ of the incident power $P_{\text {inc }}$ is absorbed and converted into heat. The ratio $P_{a b s} / P_{\text {inc }}=A_{\text {pol }}(\theta, \varphi)$, is the directional absorptivity sometimes called absorptance. $A_{\text {pol }}(\theta, \varphi)$ is function of the angle of incidence $\theta$ and of the beam polarization ( $\mathrm{pol}=\mathrm{p}$ or $\mathrm{s}$ ). For an anisotropic body it also depends on the azimuthal orientation of the sample. Absorptivity is mainly governed by the intrinsic optical properties of the sample constituants (complex optical indexes) and by morphological or geometrical parameters (roughness characteristics for non-plane surfaces, thicknesses of the layers for stratified media).

Directional absorptivity studies on non-plane surfaces are very interesting due to singular or resonant absorption phenomena which can occur : gratings anomalies, surface enhanced absorption, surface polariton excitation.... Another application of directional absorptivity measurements on rough surfaces is the determination of the directional emissivity (by virtue of Kirchhoff's law) especially at room or low temperature where conventional methods are difficult to use due to background emissivity. For opaque materials absorptivity and reflectivity are linked by the conservation law [1]:

$$
\mathrm{A}_{\text {pol }}(\theta, \varphi)+R_{\text {pol }}(\theta, \varphi)=1
$$

In this equation $R_{\text {pol }}(\theta, \varphi)$ is the directional - hemispherical reflectivity sometimes denoted $\rho_{\lambda}(\theta, \varphi)$. When reflectivity experiments are feasible, a direct measurement of $A_{p o l}(\theta, \varphi)$ is not useful as it can be deduced from Eq. (1). For structures which scatter light in a lot of directions (multiorder 1D or 2D gratings) or in a wide angular range (very rough surfaces) measurement of $R_{\text {pol }}(\theta, \varphi)$ is by no means an easy task especially at variable incidence angle. So, in these cases, a direct measurement of $A_{\text {pol }}(\theta, \varphi)$ rather than its derivation from reflectivity is necessary. To achieve that three methods are available : calorimetry [2], photoacoustic characterization [3] or photothermal deflection experiments [4]. The first two suffer from difficulties which come from the need of an adiabatic enclosure in calorimetry and of a window-cell in photoacoustics. In both case an angular limitation for the pump beam results. We choose to use Transverse Photothermal Deflection (Mirage) because it gives a large angular accessibility on the sample and, due to its high sensitivity, allows to employ a low power pump beam.

In section 2 , the connection between deflection signal and absorptivity is discussed in the framework of a simplified 1D model. The use of a variable angle pump beam is taken into account. In section 3 , the exprimental setup is described. Section 4, deals with experimental results and is divided in two parts. The first one presents an experimental validation of the 1D photothermal model. In the second one, directional absorptivity measurements obtained on a silicon 2D relief-grating are presented. Finally, these results are qualitatively analysed with the help of an electromagnetic model valid for $1 D$ gratings. 


\section{2 - DESCRIPTION OF THE METHOD}

For a 1D incident pump beam of width $W$ and uniform power repartition there is a simple linear relation between the angular variation $D(\theta)$ of the measured photothermal deflection and the directional absorptivity. As a matter of fact the diminution of the pump power density when $\theta$ increases is exactly recovered by an increase of the length of the probed heated area as shown by Fig. 1 and Eq.(2).

$$
D(\theta)=C\left(\frac{W}{\cos \theta}\right)\left(\frac{P_{\text {inc }} \cos \theta}{W}\right) A(\theta)=C P_{\text {inc }} A(\theta)
$$

$C$ is a factor which only depends on the probe beam geometrical parameters and on the thermal properties of the media.

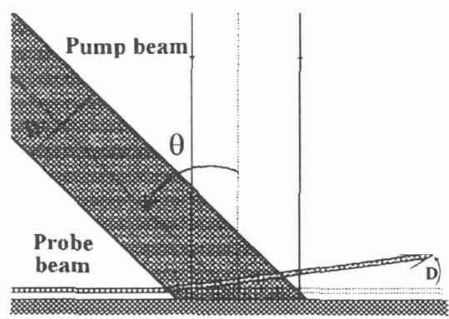

Fig. 1

When the directional absorptivity at a particular angle $\theta_{0}$ is known (for instance at normal incidence) this approach allows to determine $A(\theta)$ on a wide angular range in a very simple manner

$$
A(\theta)=A\left(\theta_{0}\right) \frac{D(\theta)}{D\left(\theta_{0}\right)}
$$

The assumption of a ID pump beam is quite unrealistic however it is shown in Sec. 3 that an expanded pump beam can achieve pump conditions which ensure Eq. (3) to be satisfied. When using a pump beam of small diameter the 1D model is no longer valid and $A(\theta)$ cannot be deduced so easily from a known value $A\left(\theta_{0}\right)$. In this case a different approach is used which allows the directional absorptivity of a rough or corrugated surface to be determined using the Fresnel absorptivity of the material as a reference. Practically one manage to have on the same sample two adjacent areas, the first one being the rough surface to be studied the second one being smooth. Then the deflections $D_{\text {rough }}(\theta)$ and $D_{\text {smooth }}(\theta)$ which corresponds to each region are measured in the very same conditions. As the thermal diffusion length is quite larger than the geometrical characteristics of the rough surface, an identical thermal behaviour is assumed for both regions. The following relation applies :

$$
\frac{D_{\text {rough }}(\theta)}{D_{\text {smoonh }}(\theta)}=\frac{A_{\text {rough }}(\theta)}{A_{\text {smouth }}(\theta)}
$$

In this equation $A_{\text {rough }}(\theta)$ and $A_{\text {smooth }}(\theta)$ are the directional absorptivities for rough and smooth regions respectively. Knowing the optical indexes of the studied material, $A_{\text {smooth }}(\theta)$ can be calculated from the Fresnel relations, allowing $A_{\text {rough }}(\theta)$ to be deduced from deflection measurement

\section{3 - EXPERIMENTAL SETUP}

$$
A_{\text {rough }}(\theta)=A_{\text {Fresnel }}(\theta) \frac{D_{\text {rriugh }}(\theta)}{D_{\text {smmiath }}(\theta)}
$$

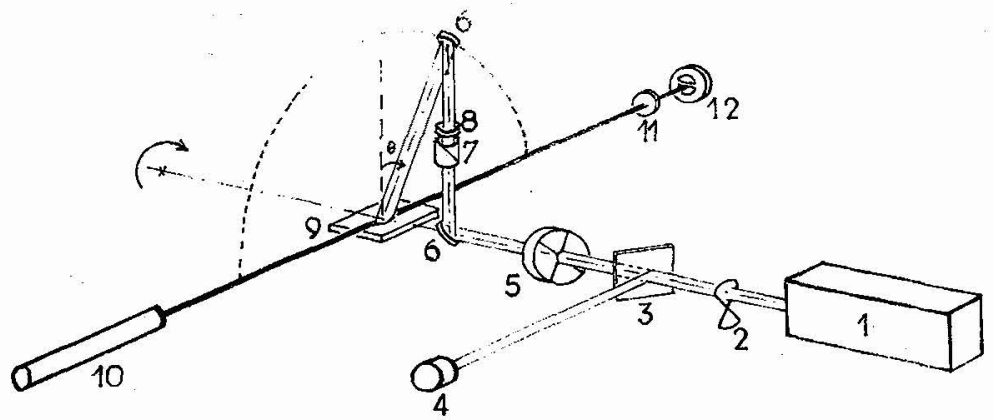

\footnotetext{
1. Pump laser 2. Chopper 3: Beam splitter 4: Detector 5. Quarter-wave plate

6: Mirrors 7: Polarizer 8: Half-wave plate 9: Sample 10 : Probe laser
}

i). Notch filter 12 : Position sensor Fig. 2 
The experimental configuration is shown in Fig. 2. For the pump beam an He-Ne or argon laser is used. The beam intensity is periodically modulated by a mechanical chopper. A beam splitter allows a reference beam to be sent onto a detector for the purpose of power normalization. The probe beam is a nonfocused He-Ne laser of about $1 \mathrm{~mm}$ diameter. Photothermal deflection is measured by a two-quadrant position sensor in fromt of which a notch filter is placed to avoid stray light. Conventional lock-in technique is used for signal detection. A rotating mechanical device supporting two mirrors (annotates 6 in Fig. 2) allows the pump beam to be directed at variable incidence angle onto a definite point of the sample. Available incidence angles range from $-80^{\circ}$ to $+83^{\circ}$. As $\theta$ increases the pump beam spot on the sample becomes more and more elliptic. Great care is taken to keep aligned the probe beam with the ellipse major axis. A definite polarization ( $p$ or $s$ ) of the beam on the sample is obtained by the combination of a prism polarizer and an half-wave plate. Prior to the first mirror a quarter-wave plate is used to transform the linear polarized wave coming from the pump laser into a circular one. So, the light intensity reflected by this mirror does not change markedly as $\theta$ varies. It remains however a residual angular variation of the incident power It is recorded and taken into account during the photothermal measurements

\section{4 - RESULTS \\ 4-1 Experimental validation}

A preliminary task was (i) to confirm that photothermal deflection measurements with a pump at variable angle of incidence give confident information about directional absorptivity (ii) to check the validity of Eq. (3). For that purpose a smooth silicon sample of well known optical index was studied. In the experiment the pump was an He-Ne laser $(\lambda=632.8 \mathrm{~nm})$ of $10 \mathrm{~mW}$. A chopping frequency of $30 \mathrm{~Hz}$ was used. To achieve an heated area which roughly corresponds to a $1 \mathrm{D}$ situation, an expander giving a beam of $12 \mathrm{~mm}$ diameter was employed. Photothermal deflection angular signatures $D_{\text {pol }}(\theta)$ were measured with $\theta$ varying from 0 to 80 degrees. The two cases of polarization were considered Fig 3 gives the results after normalization by $D(0)$ the deflection obtained with the pump at normal incidence. For comparison Fig. 3 also shows $A_{\text {pol }}(\theta) / A_{\text {pol }}(0)$ the normalized Fresnel absorptivities calculated taken $\mathrm{n}^{*}=3.88+$ i0.02 for the optical index of silicon at $632.8 \mathrm{~nm}$. The fairly good agreement observed between measured and calculated values gives an overall confirmation of our approach. Also emphasized is the extremly low incident pump power, about $3 \mathrm{~mW}$, employed.

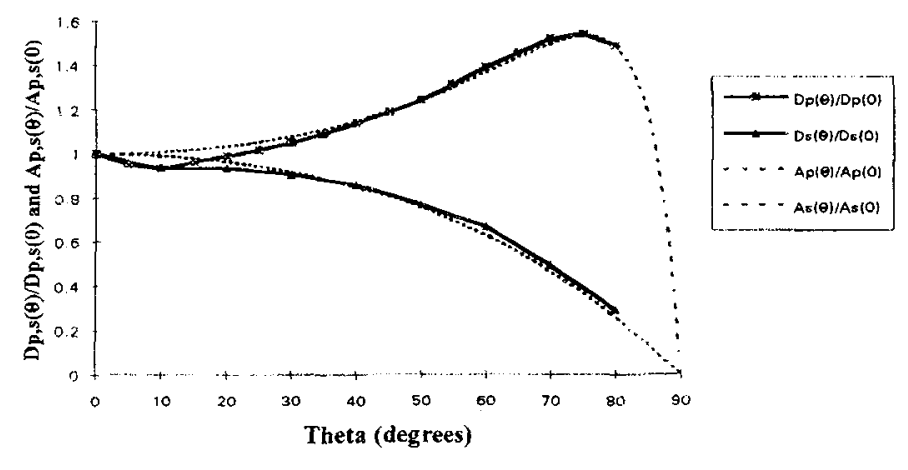

Fig.3

\section{4-2 Application to a material with a non-plane surface}

\section{4-2-1 Results}

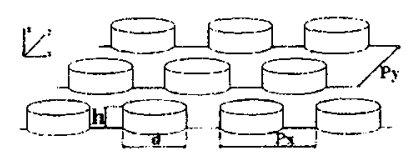

Fig. 4

As discussed in the introduction directional absorptivity measurements are specifically useful in the case of scattering samples. To illustrate the capability of the technique a $2 \mathrm{D}$ surface-relief silicon grating was studied. The 2D grating was frabricated by reactive ion etching. It consists in a square lattice (period $=5 \mu \mathrm{m}$ ) of cylindrical pillars (fig. 4 ). The height $h$ and diameter $d$ of the pillars are $0.4 \mu \mathrm{m}$ and $2 \mu \mathrm{m}$ respectively. In this experiment the pump beam was delivered by an argon laser at $514.5 \mathrm{~nm}$. A chopping frequency of $827 \mathrm{~Hz}$ was used. The incident power on the sample was about $50 \mathrm{~mW}$. Position of the $2 \mathrm{D}$ grating is such that one of its primitive vectors is parallel to the plane of incidence, the other being perpendicular. As explained in Sec. 2 , two adjacent regions coexist on the sample. On the smooth one, specular Fresnel reflection occurs. The other includes the $2 \mathrm{D}$ grating and scattering over several hundreds of diffraction orders takes place. Photothermal deflection signatures $\mathrm{D}(\theta)$ are recorded in exactly the same condtions for each region. Fig. 5 presents the results for both polarization It can be shown that silicon absorptivity is significantly 
modified by the 2D grating At low incidence angles an increase of a few percents is observed whatever the polarization. At high incidence angles pabsorptivity becomes smaller than for the smooth case while s-absorptivity drastically increases and shows a clear singularity at about 64 degrees.

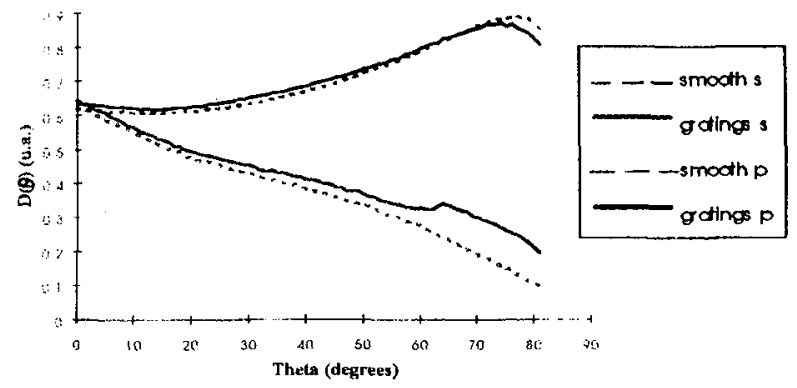

Fig. 5

\section{4-2-2 Discussion}

Due to the high amplitude (h-0.4 $\mu \mathrm{m}$ ) of the $2 \mathrm{D}$ grating as compared to the wavelength, a qualitative discussion is questionable. A numerical simulation is clearly needed. Such an approach is in progress in our lab. Up to now only ID gratings with low-slope profile can be treated. Basically the method uses a plane-wave expansion to describe the field at the grating surface (Rayleigh method) [5]. For a given incidence angle and a given polarization, the efticiencies $\mathrm{e}_{\mathrm{pol}}(\theta)$ of the various diffraction orders can be calculated. Then the directional absorptivity is given by

$$
\mathrm{A}_{\mathrm{pol}}(\theta)=1-\sum_{\text {all orders }}^{\mathrm{pol}} \mathrm{e}_{\mathrm{pol}}^{(\mathrm{m})}(\theta)
$$

In spite of the model inadequacy we tentatively compared our experimental results with the calculated absorptivity given by this $1 \mathrm{D}$ simulation in the case of a sinusoidal silicon grating of period $5 \mu \mathrm{m}$ and total amplitude $0.4 \mu \mathrm{m}$. For the calculation $n^{*}=4.24+\mathrm{i} 006$ was used as the silicon optical index at $514.5 \mathrm{~nm}$. Only the s polarization case is considered here Fig. 6 shows the result. It can be seen that $D_{s}^{\text {gating }}(\theta) / D_{s}^{\text {smooth }}(\theta)$ exhibits a similar behavior as the calculated quantity

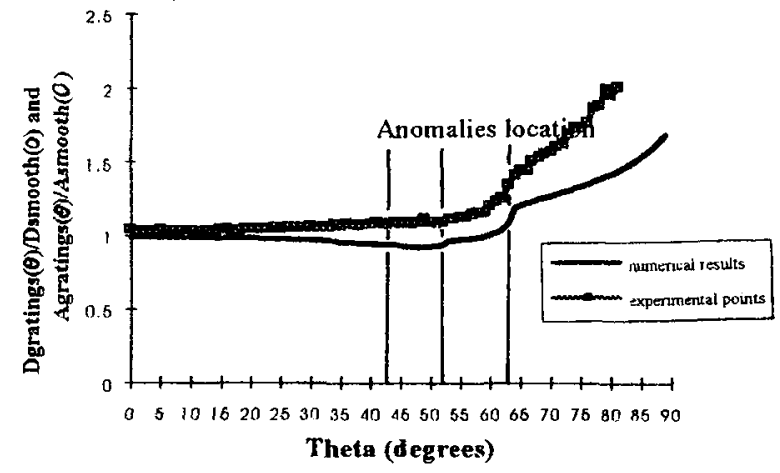

Fig. 6 $\mathrm{A}_{\mathrm{s}}^{\text {gating }}(\theta) / \mathrm{A}_{\mathrm{s}}^{\text {smooth }}(\theta)$. It is worthwhile noticed that the same singularities appear on both curves. In fact these singularities correspond to grating anomalies which are due to the passing off of diffraction orders through the grazing angle. These anomalies occur [6] at angles given by :

$$
\theta=\operatorname{Sin}^{-1}\left(1-m \frac{\lambda}{p}\right) \quad m=0,1, \ldots
$$

From these preliminary results it can be concluded that directional absorptivity measurement by photothermal deflection allows singular absorption phenomena on $2 \mathrm{D}$ gratings to be studied quite easily.

The authors would like to thank Stéphane Mainguy for assistance in numerical computation by the Rayleigh method.

\section{References :}

[1] - J. Taine and J.P. Petit, Transferts thermiques (Dunod 1989).

[2] - T. J. Wieting and J.L. De Rosa, J. Appl. Phys 50, 1070 (1979)

[3] - T. Inagaki, J.P. Goudonnet, J.W. Little and E.T. Arakawa, J. Opt. Soc. Am. B 2, 433 (1985).

[4] - A.C. Boccara, D. Fournier and J. Badoz, Appl. Phys. Lett. 36, 130 (1980).

[5] - A. A. Wirgin, Optica Acta 28, 1377 (1981)

[6] - A. Hessel and A. A. Oliner, Appl Opt 4, 1275 (1965). 